\title{
2021 Breakthrough Prizes Announced
}

\section{Breakthrough Prize in Mathematics}

The Breakthrough Prizes honor important, primarily recent, achievements in the categories of fundamental physics, life sciences, and mathematics.

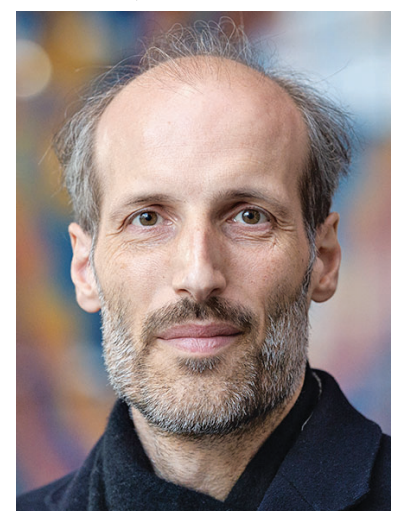

Martin Hairer

Martin Hairer of Imperial College London has been awarded the 2021 Breakthrough Prize in Mathematics "for transformative contributions to the theory of stochastic analysis, particularly the theory of regularity structures in stochastic partial differential equations."

Hairer, who was awarded the Fields Medal in 2014, was born in Geneva, Switzerland. He received his $\mathrm{PhD}$ from the University of Geneva in 2001 under the supervision of Jean-Pierre Eckmann. Before joining Imperial College, he held appointments at the University of Warwick and the Courant Institute of New York University. In addition to the Fields Medal, his honors include the 2008 Whitehead Prize of the London Mathematical Society (LMS); the Philip Leverhulme Prize (2008) and Leverhulme Research Leadership Award (2012), both from the Leverhulme Trust; the Wolfson Research Merit Award of the Royal Society of London (2009); the Fermat Prize (2013); and the Frölich Prize of the LMS (2014). He was elected a Fellow of the Royal Society in 2014 and has been honored with the award of Knight Commander of the Order of the British Empire. Hairer is a Fellow of the AMS.

Hairer tells the Notices: "Both my wife, Xue-Mei Li, and my father, Ernst Hairer, are mathematicians (at Imperial College London and retired from the University of Geneva, respectively). I do some coding in my spare time. In particular, I have been developing the sound editor Amadeus

For permission to reprint this article, please contact: reprint-permission aams.org.

DOI: https://dx.doi.org/10.1090/noti2206 for a very long time (since the end of high school in the early nineties). While I don't have all that much spare time to spend on it nowadays, I do still maintain it and make sure that it runs smoothly on the most current versions of macOS."

The Breakthrough Prizes are sponsored by Sergey Brin, Priscilla Chan and Mark Zuckerberg, Ma Huateng, Jack Ma, Yuri and Julia Milner, and Anne Wojcicki. The prize carries a cash award of US\$3 million.

\section{New Horizons in Mathematics Prizes}

Three New Horizons in Mathematics Prizes were also awarded for 2021 to promising early-career researchers. The prizes carry a cash award of US\$100,000. The prizes were awarded to the following.

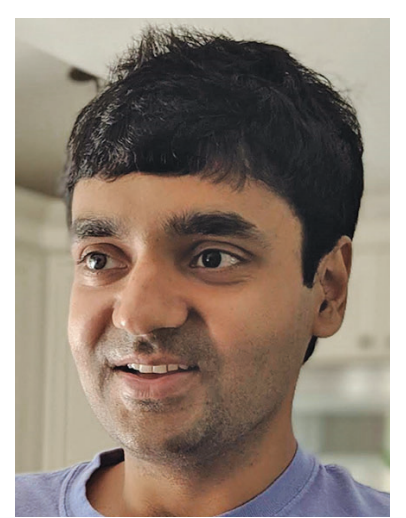

Bhargav Bhatt
Bhargav Bhatt of the University of Michigan was honored "for outstanding work in commutative algebra and arithmetic algebraic geometry, particularly on the development of $p$-adic cohomology theories." Bhatt received his $\mathrm{PhD}$ from Princeton University in 2010 under the direction of Aise Johan de Jong. He joined the University of Michigan in 2010, where he is now full professor. He spent 2012-2014 on leave at the Institute for Advanced Study as a member in the School of Mathematics. He received a Packard Fellowship for 2015 through 2021. He was awarded the Compositio Prize in 2016. He held the Eilenberg Chair at Columbia University in the fall of 2018 and the Chern Professorship at the Mathematical Sciences Research Institute in spring 2019. He has been named a Simons Investigator for 2019 through 2024. 


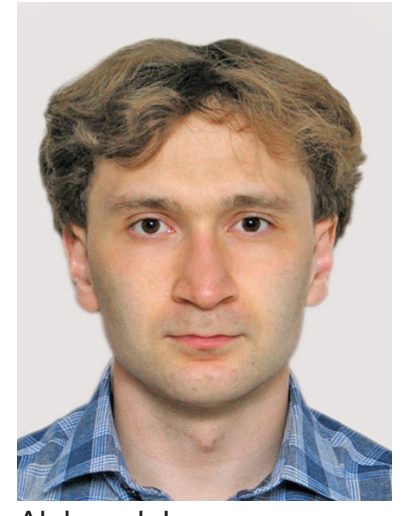

Aleksandr Logunov
Aleksandr Logunov of Princeton University received a New Horizons Prize "for novel techniques to study solutions to elliptic equations and their application to long-standing problems in nodal geometry." Logunov received his $\mathrm{PhD}$ in 2015 from St. Petersburg State University under the direction of Viktor Havin. He has been a junior research fellow at Chebyshev Laboratory, St. Petersburg State University; a postdoctoral fellow at Tel Aviv University; and a research scholar (2017-2018) at Princeton, where he is now assistant professor. In 2017 he received a Clay Research Award jointly with Eugenia Malinnikova. He received the St. Petersburg Mathematical Society Prize and the Moscow Mathematical Society Prize, both in 2017. In 2018, he was named a Clay Research Fellow and was awarded the Salem Prize. He received a Packard Fellowship in 2019 and the EMS Prize of the European Mathematical Society in 2020. He was an invited speaker at the International Congress of Mathematicians in Rio de Janeiro, Brazil, in 2018.

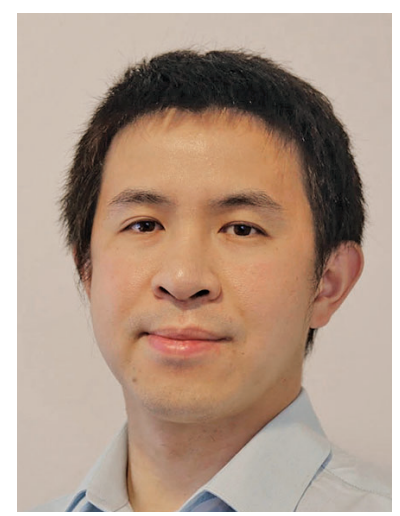

Song Sun

Song Sun of the University of California, Berkeley, was honored "for many groundbreaking contributions to complex differential geometry, including existence results for Kahler-Einstein metrics and connections with moduli questions and singularities." Sun received his $\mathrm{PhD}$ from the University of Wisconsin in 2010, advised by Xiuxiong Chen. He has been a research associate at Imperial College London and an assistant professor at Stony Brook University. He joined the faculty at Berkeley in 2018, where he is an associate professor. His honors include a Sloan Foundation Research Fellowship and the Veblen Prize in Geometry (with Xiuxiong Chen and Simon Donaldson) in 2019. He was also an invited speaker at the International Congress of Mathematicians in Rio de Janeiro in 2018. He tells the Notices: "I have one hobby-playing badminton. I have been regularly doing it for about fourteen years, but I have unfortunately stopped playing for six months for obvious reasons, and I hope I will be able to continue this safely in the near future."

\section{Maryam Mirzakhani New Frontiers Prizes}

The inaugural Maryam Mirzakhani New Frontiers Prizes were awarded to three early-career women whose work involves the mathematical sciences.

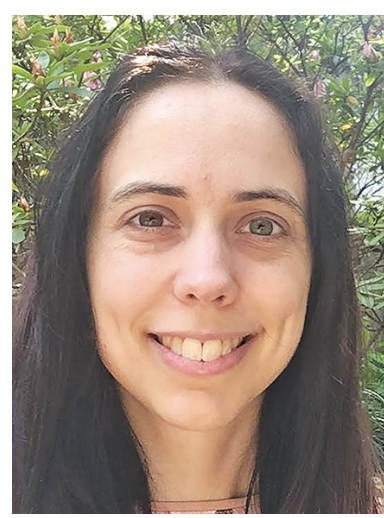

Nina Holden
Nina Holden of ETH Zurich was honored "for work in random geometry, particularly on Liouville quantum gravity as a scaling limit of random triangulations." Holden received her PhD from the Massachusetts Institute of Technology in 2018 under the direction of Scott Sheffield. She is a Junior Fellow at ETH Zurich, working with Wendelin Werner. In 2021, she will join the faculty of the Courant Institute of Mathematical Sciences as an associate professor. Her honors include the SwissMPA Innovator Prize (2019) and a Bernoulli Society New Researcher Award (2020). She is coorganizer, with Ewain Gwynne and Xin Sun, of an online seminar on Random Geometry and Statistical Physics. She tells the Notices: "I grew up in Norway, and in my spare time I like to do cross-country skiing."

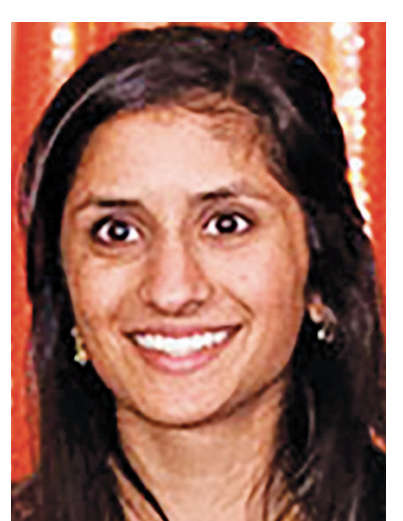

Urmila Mahadev
Urmila Mahadev of the California Institute of Technology was honored with a New Frontiers Prize "for work that addresses the fundamental question of verifying the output of a quantum computation." She received her $\mathrm{PhD}$ from the University of California, Berkeley, in 2018. She received the Machtey Award at the 2018 Symposium on Foundations of Computer Science for her work on verification of quantum computing. She expects to concentrate her future research efforts on exploring problems in the intersection of theoretical computer science and quantum computing.

Lisa Piccirillo of the Massachusetts Institute of Technology received a New Frontiers Prize "for resolving the classic problem that the Conway knot is not smoothly 'slice.'" She received her $\mathrm{PhD}$ from the University of Texas at Austin in 2019 under the direction of John Luecke. Her specialty is the study of three- and four-dimensional spaces. She was awarded an NSF Postdoctoral Research Fellowship for 2019-2020. She tells the Notices: "There was a summer in graduate school during which I bought and repaired two '70s-era Japanese motorcycles, and at the end of which I failed all my prelim exams. I love to make things with my 


\section{COMMUNICATION}

hands, and I am rarely prouder of myself than when I can make something instead of buying it."

The Maryam Mirzakhani New Frontiers Prize was established in 2019 and named for the famed Iranian mathematician, Fields Medalist, and Stanford professor who passed away in 2017. During her exceptionally prolific career, Mirzakhani made groundbreaking contributions to the theory of moduli spaces of Riemann surfaces. The New Frontiers Prize award of US\$50,000 will be presented annually to women mathematicians who have completed their PhDs within the previous two years.

_Elaine Kehoe

\section{Credits}

Photo of Martin Hairer is courtesy of Imperial College London.

Photo of Bhargav Bhatt is courtesy of Katherine Grillaert. Photo of Nina Holden is courtesy of Nina Holden.

Photo of Urmila Mahadev is courtesy of Urmila Mahadev. 